\title{
A Study on Various Mesh Generation Techniques used for Engineering Applications
}

\author{
${ }^{1}$ T.V. Smitha, ${ }^{2}$ Madhura S, ${ }^{3}$ Sindhu R, ${ }^{4}$ Brundha R \\ ${ }_{1,2,3,4} \mathrm{RV}$ Institute of Technology and Management, Bangalore, India \\ ${ }^{1}$ smithatv.rvitm@rvei.edu.in \\ ${ }^{2}$ madhuras.rvitm@rvei.edu.in \\ ${ }^{3}$ sindhurm_ec19.rvitm@rvei.edu.in \\ brundhar_ec19.rvitm@rvei.edu.in
}

\begin{abstract}
In this paper our aim is to provide a survey of mesh generation techniques for some Engineering applications. Mesh generation is a very important requirement to solve any problem by very popular numerical method known as Finite element method (FEM). It has several applications in various fields. One such technique is Automated generation of finite element meshes for aircraft conceptual design. It's an approach for automated generation of fully connected finite element meshes for all internal structural components, given wing body, geometry model, controlled by a few conceptual level structural layout parameters. Another application where it is used is in the study of biomolecules to generate volumetric mesh of a biomolecule of any size and shape based on its atomic structure. These methods are proved to be a faster method due to the usage of computing techniques. Mesh generator is also used for creating finite element surface and volumetric mesh from 3D binary and gray scale medical images. Some of the applications include volumetric images, surface mesh extraction, surface mesh repairing and many more. It is of great importance in understanding the human brain which is a complex subject. Though $3 \mathrm{D}$ visualization is a useful tool available, yet it is inadequate due to its challenging computational problem. This paper also includes the survey on latest tools used for these applications which overcomes many problems associated with the conventional approaches.
\end{abstract}

Keywords- Mesh Generation, Finite Element Method, Biomolecules, 3D Visualization 


\section{INTRODUCTION}

Biomolecular structure determines its function is the basic principle of Molecular Biology. Deficiencies and deformities in biomolecular structure causes diseases and syndromes. So, the study of biomolecular structures and their physicochemical properties becomes important [1]. Many tools are available to study biomolecular complexes viz atom models and do not provide results when studied biomolecule complex sizes which are greater than $10^{\wedge} 5$ atoms. On the other hand, Molecular coarse grain model works well in such cases, yet they too have upper limits due to lack of current computational tools. The volumetric meshes overcome these deficiencies and they are compatible with numerical methods. E.g.: - FEM which helps in performing simulation of biophysical processes. In this paper we describe the development of an algorithm design which generates a volumetric mesh which can be applied to major categories of biomolecules [2]. Understanding the brain and its structure and functions are a big challenge to the neuro scientist's due to its complexity. Though 3D Visualization by using $3 \mathrm{D}$ polygonal mesh which approximated cell anatomy is a useful tool for evaluation of complex system. Vast numbers of neurons with its complex and complicated geometry, makes Visualization difficult and computation costly. In this paper we present the new tool NeuroTessMesh, which is designed to overcome many a problem faced by Visualization techniques of neural circuit [3].

In recent times, the medical researchers have been focusing on multi-modal imaging because of its ability to combine structural and functional imaging modalities and due to its acceptance by new imaging modalities. Here, before producing functional image, we have to create a modal-based image, which can be done using numerical methods, of which FEM turned out to be the choice of many. The most important task and pre-requisite in it is meshing. There are many algorithms and models to implement it, but here we'll be discussing about the very efficient one, which is formation of triangular surfaces and tetrahedral meshes. Next comes FEA used for a full aircraft, which is time consuming and requires a lot of labour [4]. The automation process available now during conceptual design of aircraft is basically for individual components. Using algorithms, each internal structure component is designed and then the finite element meshes that are fully connected is generated automatically. These can be produced virtually too using many software. But, putting these components together has few 
Journal of Innovative Image Processing (JIIP) (2021)

Vol.03/ No. 02

Pages: $75-84$

https://www.irojournals.com/iroiip/

DOI: https://doi.org/10.36548/jiip.2021.2.001

challenges. The idea here is to form a model in which two surfaces connected share a common boundary curve and performs automated generation of finite element mesh that is fully connected. It has many processes starting from specifying parameters at conceptual level for internal components to design optimization [5].

\section{CASE STUDY 1}

Meshing techniques are basically of three types: Advancing front (ADF), Delaunay Triangulation (DT) and Space Decomposition (SD). The ADF technique is not feasible due to its complexity and time consumption. While DT's major drawback is, it does not store the memory of the mesh domain. Whereas the SD technique is most preferred due to its short time consumption and high parallelizable methods. That is why it is widely used by FEM researchers.

There are two types of SD methods, viz Bin and Octree methods, which create FEM suitable meshes. Though both the algorithms look similar, the bin technique divides bounding box which requires excessive memory. Whereas the octree technique performs divisions based on the domain to be meshed [6]. The main advantage of SD method is creation of many meshes due to its adaptability. While its major drawback is its inability to preserve the sharp features of the meshes.

\section{a) Octree Meshing:-}

In this technique subdivisions are created so as to generate Octree cells with different size. These cells can be used to form tetrahedral meshes [7]. In order to create tetrahedral meshes all the elements should be of same size, which is already present in this mesh. It's important for the Octree cells to be represented in binary number, as bitwise operations are faster than compared to arithmetic operations. During the subdivision of Octree, the selection of cells becomes important. Some of the features the cells must have in order to be a part of final mesh are: -

- Two distinct elements must not overlap.

- The elements must be positively oriented. 
- In $2 \mathrm{D}$, an edge is only shared by two adjacent elements, in $3 \mathrm{D}$, a face is only shared by two adjacent elements.

b) Capsid mesher:-

There are many methods to mesh a capsid but each of them has major drawbacks. Among these the best method proposed is Octree method due to its short time consumption on generating the capsid mesh [8].

\section{c) Nanoindentation:-}

In this paper the experimental results of the numerical simulation of the nanoindentation of the whole viral capsid, using volumetric meshes is compared with that of [4] for the CCMV. And the result shows the indentation were almost identical in both the algorithms. However significant difference occurs when the mesh resolution is increased. Whereas in Octree computing technique its parallel implementation plays an important role in reducing the time consumption compared to CapsidMesh process.

\section{CASE STUDY 2}

The main aim of NeuroTessMesh is to show an efficient representation of the complex neural structure of the brain along with accurate results which matches the experimental data. This paper proposes a set of techniques which can be grouped into two modules [9]. The first module takes the input of the neurons existing structure and generates a course low-poly-3D mesh along with some additional information. The second module takes the course generated mesh with the additional information and refines it.

\section{a) Generation module:-}

The first step in this module is to generate an initial low-poly mesh, which approximates the whole neuron. The tracing which is required for this module are taken from existing NeuroMorpho which describes the soma and neutrites in different ways. Since both are different, separate meshes are generated which are merged in the final step. To generate the mesh for somata a sphere is created by simulating physical deformation. This is done so that 
Journal of Innovative Image Processing (JIIP) (2021)

Vol.03/ No. 02

Pages: $75-84$

https://www.irojournals.com/iroiip/

DOI: https://doi.org/10.36548/jiip.2021.2.001

the neutrites can attract the sphere towards it, thus resulting in elastic deformation of the sphere. To simulate this deformation FEM is used as an improved technique over that of [5].

FEM works on volumetric models. So the first step is to create volumetric representation of a poly sphere made of triangles of each soma. Morphological tracings provide us with the radius and soma center. Based on this tetrahedral mesh is built. This mesh is taken as an initial equilibrium state for an elastic deformation process. The ecosphere and the surface of tetramesh has triangular shapes. But these triangular shapes are not suitable for this method. Hence, a quad shape is created by combining two adjacent triangles. The neutrite present in the soma pull the quads which are nearest to them, thus resizing the quad to match the neutrite diameter at its starting point. Finally, FEM is applied to the mesh, such that the soma is deformed.

Regarding formation of mesh of neutrite, orientation vector needs to be computed. In this computation there are three different stages:

- Standard tracing point

- Bifurcation

- Ending tracing point.

Using the vectors $\mathrm{r} 0$ and $\mathrm{r} 1$ we can find orientation vector $\mathrm{o}$. Once orientation vectors have been computed, a section quad is aligned to the orientation vector. Finally, neutrite quad mesh is formed by combination of all section-quads of neurite [10]. The final generated polygon which is the combination of coarse neurite and soma can approximate the cell membrane which will be refined in the second module. The use of additional information helps as a guide to the refinement step.

\section{b) Refinement module:-}

In this module we aim to approximate high resolution neuron membrane. Regarding soma, the resolution is obtained by elastic deformation whereas neurite the fly-refinement procedure with tessellation capabilities is performed. In this tessellation process there are three substages [11]. The first substage includes determining the subdivision levels for each patch. The second substage divides the original patch accordingly. Finally, in the third substage, accurate 
Journal of Innovative Image Processing (JIIP) (2021)

Vol.03/ No. 02

Pages: $75-84$

https://www.irojournals.com/iroiip/

DOI: https://doi.org/10.36548/jiip.2021.2.001

positions are generated with the help of previous substages. Hence the refinement process is complete [12].

\section{ANALYSIS}

The NeuroTessMesh tool efficiently approximates the cell bodies, the dendritic and axonal arbors. Triangular and tetrahedral imaging. There are types of image-based mesh generations depending on the applications. This process involves of breaking the mesh generation task into atomic sub-tasks which can later be used independently or together based on applications. The mesh generation work flow is made up of connections, where each connection represents an atomic subtask which is involved in conversion of image into the target state [13]. It mainly consists of two steps, the first one being generation of triangular iso surfaces of specific density of given input 3D image. Certain procedure is carried out to convert the non-closed iso surface to a closed one, later in order to remove duplicated elements, certain mesh repairing process is performed [14]. Second step involves to fill the previously obtained triangular iso surfaces with tetrahedral elements. As for the input, any 3D binary image can be fed into this mesh generator. Mesh extraction of this surface can be done either by surface simplification (SS) which involves generation of a triangular mesh boundary followed by mesh simplification process or Constrained Delaunay tetrahedralization (CDT) approach which involves direct generation of triangular surfaces. In order to continue with process of tetrahedral mesh generation, we have to get rid of deficiencies like duplicated triangles, isolated vertices using an external tool and this is called surface mesh repairing [15]. The subsequent process is sub region labeling and hole specifications here the important aspect is to find a specific interior point for each subregion. But this process is not easy as the centroids not necessarily be located inside the domain as surfaces may not particularly be convex. Therefore, we have to determine interior points such that they are placed at a reasonable distance from boundary using distance field method where all voxels are set between 0 and 1, later use Gaussian smoothening and produce a 3D field by repeating process for $\mathrm{N}$ times. We can later mask results by each image segment and make sure points are strictly located inside specified subregions. The hollow regions can be tagged and with application of same algorithm. Interior points can be determined and later removed from final tetrahedral mesh using regional labels. It later involves process of creating 
Journal of Innovative Image Processing (JIIP) (2021)

Vol.03/ No. 02

Pages: $75-84$

https://www.irojournals.com/iroiip/

DOI: https://doi.org/10.36548/jiip.2021.2.001

water tight surfaces. The final step being the smoothing of surface mesh using 3 algorithms to optimize the quality of surface mesh.

The results have been proved by taking a tissue of complex vessel network and human brain MRI image consisting of gray and white matter.

\section{a. Automated FEA Process}

Now, let us get to know the numerical methods included to make a fem-ready geometry, to generate finite element meshes. The basic idea of the automation processes includes of gluing the individually prepared internal structural components of aircraft constructed using finite element meshing. But here, a FEM ready geometry is created using several meshing tools where our sole concentration is about connecting various components and let the meshing tools take care with problems related to meshing. In a FEM ready geometry represented in rectangular form, the infeasible layout parameters are automatically detected and can be corrected when spar location and wing shape are depicted. If feasible, fully connected surfaces for internal structural components and skins is constructed which consists of triangles and quadrilaterals. Else Geompack++ can be used to do this. Later we convert all these surfaces into water tight compartments.

b. Next comes the automated finite element meshing

The present automation process makes use of PATRAN'S HYBRID mesher to generate FEM ready geometry which consists of a few triangles and Geompack++ which generates a fully connected quadrilateral mesh. For a high-resolution mesh, both the above tools might fail. PCL commands control the PATRON meshing process and FEM ready geometry surfaces are divided into 16 groups which will be exported to 1 IGES file consisting of many bilinear Bspline surfaces, where surfaces with $y>0$ or $y=0$ are exported and all surface meshes are created. Later with usage of PATRAN'S equivalence function call, all unwanted nodes are eliminated and resulting mesh is fully connected. Later the meshes for different predefined groups are exported separately and retagged to maintain the actual FEM ready geometry. The material properties assignment, the design variable definitions and thickness design variables of all shell element is taken care. Then Geompack++ reads 3D region format and generates a quadrilateral 
Journal of Innovative Image Processing (JIIP) (2021)

Vol.03/ No. 02

Pages: $75-84$

https://www.irojournals.com/iroiip/

DOI: https://doi.org/10.36548/jiip.2021.2.001

mesh using control parameters which contains original FEM ready geometry data structure and then removing quadrilateral in half space $(\mathrm{y}<0, \mathrm{y}=0)$ is taken care in post processing [16]. Later comes process of thickness optimization where problems on thickness makes use of two constant pressures which is determined using NASTRAN bulk and PLOAD to solve it. After making all the initial guesses for the design, the NASTRAN computes optimal thickness distribution after a bulk file for NASTRAN 200 solution is automatically generated for the required thickness optimization problem. This process keeps in mind minimum user input requirements, versatility, robustness and shows an example of type of information required to run it using various meshing components. The most time-consuming step of the process is defining the conceptual level internal structural layout parameters with wing box location, spar locations [17]. The numerical verification is provided to check its versatility and robustness using a subsonic and supersonic aircraft. While calculating, the maximum relative error for optical structural weight is approximately $4 \%$ and maximum deviation is approximately $11 \%$ to $16 \%$ of optical weight of final structural mesh and it includes five meshes which is distributed by maximum displacement for a given thickness and it is proportional to PATRAN mesh size [18]. Also, maximum displacement is $1.1 \%$ for these meshes.

\section{CONCLUSION}

The volume and position of biomolecule atom produce volumetric mesh in this computational algorithm. Octree method discretize space, while parallelization technique reduces running time and enhance memory. Since, mesh elements are tetrahedral, so volumetric mesh of any shape and size can be produced and they are FEM compliant. Here a set of domain specific techniques are provided in this paper for generation, visualization of neuronal scenes, approximation of anatomy of the cells and reconstruction of neuron models stored in NeuroMorpho. Morphological tracing can help bridge the gap between neuro science and computer graphics. The above methods used for meshing can be applied in wide region viz medicine, aircraft design, etc. As the medical research presently are emphasizing more on multi modal imaging off late, the mentioned methods would help in minimizing the post processes and use less time by using available mesh techniques. It also proves it's efficiency by giving examples of tissue of complex vessel network, human brain MRI, etc. Next method tells us the 
way of meshing aircrafts at a stage of conceptual design using various features by giving a minimum input information. It involves the concept of fully connected finite element meshes and tells ways of optimizing solutions of thickness, etc. by taking into account of 20 meshes for four structural layouts with 11-16\% differences in optimal structural weight and numerical discrepancy up to $4 \%$ in optimal structural weight. But as of now, it is used only for fuselage and wing which will further cover horizontal and vertical tails, engines, etc. Therefore, these methods will give us a way to perform meshing that would require very less or only necessary post-meshing process within less time with robustness, versatility and less labor intensive.

\section{REFERENCES}

[1] [Yu2008] Yu Zeyun, Holst Michael J, Cheng Yuhui and McCammon J. Andrew Feature preseving adoptive mesh generation for molecular shape modelling and simulation Journal of molecular graphics and modelling 2008.

[2] [Cheng2009] Cheng Ho-Lun and Cshi Xinwey Quality mesh generation for molecular skin surface using restricted union of balls Journal of computational geometry theory and applications, 2009.

[3] [Vargas2012] Vargas Felix Miguel and Botello Rionda Salvador FEMT, Open Source Tools for Solving Large Systems of Equations in Parallel Acta universitaria, 2012.

[4] [Alonzo 2018] Alonzo- Velazquez Jose, Botello Rionda Salvador, Herrera Guzman Rafael and Carrillo- Tripp Mauricio. CapsidMesh: atomic- detail structured mesh representation of icosahedral viral capsid and the study of their mechanical properties. International journal for Numerical Methods in Biomedical Engineering 34:e2991, 2018.

[5] Erleben, K, Sporring, J, Henriksen, K, and Dohlman,K.(2005). Physics-Based Animation(Graphics Series). Rockland, MA: Charles River Media Inc.

[6] Boschiroli, M, Funfizig, C, Romani, L, and Albrecht, G(2011). Technical section: a comparison of local parametric CO Bezier interpolants for triangular meshes. Comput. Graph. 35,20-34. doi: 10.1016/j.cag.2010.09.011

[7] Abujar, P, Sousa, M, and Szucs, P(2013). Versatile morphometric analysis and visualization of the three-dimensional structure of neurons. Neuroinformatics 11, 393-403. doi:10.1007/s12021-013-9188-z 
Journal of Innovative Image Processing (JIIP) (2021)

Vol.03/ No. 02

Pages: $75-84$

https://www.irojournals.com/iroiip/

DOI: https://doi.org/10.36548/jiip.2021.2.001

[8] Bindhu, V., and Villankurichi Saravanampatti PO. "Semi-Automated Segmentation Scheme for Computerized Axial Tomography Images of Esophageal Tumors." Journal of Innovative Image Processing (JIIP) 2, no. 02 (2020): 110-120.

[9] Sungheetha, Akey, and Rajesh Sharma. "GTIKF-Gabor-Transform Incorporated K-Means and Fuzzy C Means Clustering for Edge Detection in CT and MRI." Journal of Soft $\begin{array}{llllll}\text { Computing } & \text { Paradigm } & \text { (JSCP) 2, } & \text { no. } & 02 & \text { (2020): }\end{array}$ 119.O.Courshesne,F.Guilbault,J.Dompierre and F.Cheriet, "Adaptive Mesh Generation of MRI Images for 3D Reconstruction of Human Trunk”,Springer Berlin/Heidelberg,2007

[10] L.Rineau and M.Y vinec,"3D Surface Mesh Generation", In CGAL Editorial Board,editor,CGAL User and Reference Manual. 3.4 edition ,2008.

[11] Madhura S, "Experimental Study on The Effect of Contrast Variation for Digital Color Video Using Absolute Difference and Piecewise Mapping Techniques”, International Conference on Innovative Mechanisms for Industry Applications (ICIMIA) held in Dayanandasagar College of Engineering from 05/03/2020 to 07/03/2020, Sponsored by IEEE Bangalore chapter, Scopus Indexed, ISBN:978-1-7281-4167-1

[12] M.Attene and B.Falcidieno, "ReMesh:An Interactive Environment To Edit And Repair Triangle Meshes”,In Proc. Of Shape Modelling Int.(SMI'06),pp. 147-164,Sep.2005

[13] Madhura.S and Suresh K," A new parallel DSP hardware compatible algorithm for noise reduction and contrast enhancement in video sequence using Zynq-7020", International Journal of Computer Aided Engineering and Technology, 2020 Vol.13 No.1/2, pp.14 - 27

[14] Laughlin,T.,Corman,J.,and Macros D., "A Parametric and Physics -Based Approach to Structural Weight Estimation of the Hybrid Wing Body Aircraft", AIAA 20131082,January 2013

[15] Joe,B., “Geompack++ Meshing Operations,” Technical Report ZCS2012-01,Sep 2012 [16] de Boor,C., “A Practical Guide to Splines(Revised Edition),’'Springer,New York,2001

[17] Shakya, Subarna, and Lalitpur Nepal Pulchowk. "Intelligent and adaptive multi-objective optimization in WANET using bio inspired algorithms." J Soft Comput Paradigm (JSCP) 2, no. 01 (2020): 13-23. 